\title{
Correspondence
}

Editor: Ian Pullen

Contents: Failure to demonstrate prophylactic efficacy of dothiepin/Sex and schizophrenia: vive la différence/ The myth of suicide prevention by general practitioners/Affect as a neurobehavioural probe in the evaluation of hypofrontality/Clinical studies of the dementias/Lewy bodies and Alzheimer's disease/ Human sleep, sleep loss, and behaviour.

\section{Failure to demonstrate prophylactic efficacy of dothiepin}

SIR: The effort involved in long-term treatment studies is prodigious and it is therefore a pity that the two-year study of dothiepin, given in a dose of $75 \mathrm{mg}$ (Old Age Depression Interest Group, Journal, February 1993, 162, 175-182), suffers from errors of design and omissions of reporting which means that the authors cannot claim efficacy in prophylaxis.

There is now a considerable literature on the longterm treatment of depression and a consensus has emerged that all antidepressant treatment courses should be continued for four to six months after apparent response, and that efficacy in preventing relapse in this continuation treatment period to consolidate response should be identified separately from subsequent prophylactic efficacy in preventing new episodes of depression. It appears from this report that the authors of the dothiepin study have not entirely understood these arguments.

The ability of the study to show prophylactic efficacy was compromised by the author's decision to deliberately overlap the continuation and prophylactic treatment phases, allowing patients to enter after eight weeks of continuation treatment. Their justification was their perception of response in the elderly as being only partial. This is difficult to accept since they have used much the same criterion of response (Montgomery \& Åsberg Depression Rating Scale $($ MADRS) < 11) that has been recommended as defining the normal range in responders (Montgomery, 1990). They give a definition of relapse of one point more on the MADRS, which is eccentric, and one would like to know what the actual scores were upon relapse.
The study was of sufficient length for separate analysis of the early continuation treatment period for consolidation of response and the later prophylactic phase for preventing new episodes. The design and the choice of rating intervals precludes analysis of the conventionally accepted six-month continuation treatment period. The choice in this study is to analyse either at the three-month, or six-month assessment point. At three months there is clearly no difference between drug and placebo. However, the exact time needed for response consolidation is not known and it is quite possible that the elderly require a longer continuation treatment and, being generous, analysis at the six-month point makes sense.

At the six-month assessment point in this study, at which patients who have received eight months of continuation treatment can be compared with those for whom placebo was substituted after two months of continuation treatment, there was no significant difference in relapse between dothiepin and placebo and it is surprising that the authors do not discuss this failure to show efficacy in relapse prevention. This was, of course, a rather small study and the risk of a type II error was consequently marked. Some therapeutic effect was observed, although the difference did not reach statistical significance, and this is consistent with other data that a less than standard dose may be effective in consolidating response (Mindham et al, 1973).

In the true prophylactic phase of this study, from six months to two years, there were six new episodes in the placebo group and five new episodes in the dothiepin group which is clearly not different. There is no discussion of the similarity of the rate of recurrence of new episodes and a less than careful reader might be misled by the authors' enthusiastic reporting into imputing prophylactic efficacy to dothiepin where it is obviously not present.

A discussion of suicide attempts would have been welcome because of concerns about toxicity in overdose with dothiepin (Cassidy \& Henry, 1987). Discussion of other serious side-effects, for example convulsions, is also lacking which would be relevant since this is the reason for the frequent reluctance to 
use the full doses at which the efficacy of dothiepin was established. There is also inadequate discussion of the reasons for the more frequent withdrawals from dothiepin during the prophylactic phase. Is it possible that patients themselves recognised that dothiepin was no more effective than placebo?

There are studies that provide clear-cut evidence of the prophylactic efficacy of antidepressants in reducing the risks of new episodes of depression (reviewed by Montgomery \& Montgomery, 1992). This is not one of them.

Cassidy, S. \& Henry, J. (1987) Fatal toxicity of antidepressant drugs in overdose. British Medical Journal, 295, 1021-1024.

Mindham, R. H. S., Howland, C. \& Shepherd, M. (1973) An evaluation of continuation therapy with tricyclic antidepressants in depressive illness. Psychological Medicine. 3, 5-17.

MONTGOMERY, S. A. (1990) The methodology necessary to establish the long-term efficacy of antidepressants. In Human Psychopharmacology: Methods and Measures (eds I. Hindmarch \& P. D. Stonier). Chichester: Wiley.

— \&MONTGOMERY, D. B. (1992) Prophylactic treatment in recurrent unipolar depression. In Long-term Treatment of Depression (eds S. A. Montgomery \& F. Rouillon). Chichester: Wiley.

Academic Department of Psychiatry

Stuart A. MONTGOMery

St Mary's Hospital

Praed Street

London W2 INY

AUTHORS' REPLY: If Montgomery thinks we "have not entirely understood [the] arguments" for separation of continuation therapy from prophylaxis, he has not read our paper carefully enough. We do discuss these arguments in some detail (p. 180; paragraphs $2 \& 3$ ) and explain why we did not apply the distinction strictly to our elderly patients, as might have been appropriate for a younger group. To reiterate and amplify the point, we were dealing with a population which has been consistently shown by research to contain only about $20 \%$ of people, or less, who will not experience a further episode of major depression; whereas the remaining $80 \%$ or so are liable either to suffer from chronic affective symptoms or one or more rapid relapses. The key question, therefore, is whether - the patient's physical state permitting - treatment should ever be stopped.

Having made the presumption that our study should have been conducted according to the design he prescribes, Montgomery employs inappropriate statistical methods to analyse it as if we had conducted it that way. In spite of our explanation (p. 177; first paragraph, second column), he does not appear to have understood the error of using crosssectional analysis ( $\chi^{2}$ tests) on longitudinal data. His statements about significance have no valid statisti- cal foundation. In fact, his assertion that "analysis at the six-month point makes sense" makes no sense at all. Our Table 2 clearly shows how relapses occurred over a two-year period, and the higher incidence in the placebo group is evident. We had hoped to make it abundantly clear that $P$ values obtained from $\chi^{2}$ tests were mentioned only to underline how misleading they can be when incorrectly applied (as, alas, they so frequently are in studies of this kind). The analysis which is appropriate to longitudinal data, such as these, is to fit a survival curve. In this instance a proportional hazards model was fitted and the survival curves speak for themselves. Clearly, it would have been preferable to have a larger sample since the consequent increase in power would have made it possible to detect a smaller difference between the treatment groups but, in the event, the difference was found to be large enough for the sample size to be acceptable.

Montgomery accuses us of giving an eccentric definition of relapse of one point more than the recommended cut-off. Where does he get this idea from? There is no inconsistency between having a criterion MADRS response $<11$ and a definition of relapse $>10$ because, provided the individual scores are integers (which they are), the two sets are mutually exclusive and exhaustive. The mean MADRS score on relapse was 24.01 .

There was no discussion of suicide or serious sideeffects in the interests of brevity. There were no suicides during the trial. Nor were there serious sideeffects, which were carefully monitored throughout in accordance with international standards. However, if it were to give Dr Montgomery some peace of mind, none of the psychiatrists taking part in this trial have an 'axe to grind' about tricyclics in general, or dothiepin in particular. The important point is the maintenance of effective pharmacological treatment, without which the great majority of this group of patients can expect their relatively short remaining life-span to be blighted by continuous or recurrent illness.

The Maudsley Hospital

ROBIN JACOBY

London SE5 8AZ

Worcester College
Oxford

$O X 12 H B$

\section{Sex and schizophrenia: vive la différence}

SIR: Lewis provides an interesting review of the literature investigating the sex differences in 